\title{
Commentary: Who is who in this storm?
}

\author{
Vito Domenico Bruno, MD, PhD, and Raimondo Ascione, MD, ChM, FETCS, FRCS
}

From the Bristol Medical School - Translational Health Science, University of Bristol, Bristol, United Kingdom. Disclosures: Authors have nothing to disclose with regard to commercial support.

Received for publication Nov 28, 2018; accepted for publication Nov 28, 2018; available ahead of print Jan 17, 2019.

Address for reprints: Raimondo Ascione, Bristol Medical School-Translational Health Sciences, University of Bristol, Research Floor Level 7, Bristol Royal Infirmary, Upper Maudlin St, Bristol BS2 8HW, United Kingdom (E-mail: R.Ascione@bristol.ac.uk).

J Thorac Cardiovasc Surg 2019;158:406-7

$0022-5223 / \$ 36.00$

Crown Copyright (c) 2018 Published by Elsevier Inc. on behalf of The American Association for Thoracic Surgery https://doi.org/10.1016/j.jtcvs.2018.11.115

Cardiac surgery is an effective, evidence-based clinical practice built on decades of research spanning from basic science to rigorous large animal studies and randomized controlled trials (RCTs) in patients. This translational approach has benefited patients enormously. ${ }^{1}$ More recently, we are witnessing a surge of different research methods from bench-based simulation to overnight observational studies from registries and meta-analysis that are raising questions and debates. ${ }^{2}$ Concomitantly, in the era of innovation, we witness a crisis in research reproducibility, ${ }^{3}$ limited reporting of registered $\mathrm{RCTs},{ }^{4}$ and a call to resurface rigorous large animal research. ${ }^{5}$ One is left wondering if these are signs of a perfect storm. In these new times, the typical cardiac surgeon looks like just a passenger on the boat of innovation, struggling to keep the rudder straight while sailing across the troubled waters of intellectual property, commercialization, conflict of interests, harm, ${ }^{6}$ lack of evidence, ${ }^{7}$ visibility, patient interest, and evidence-based medicine. Who is who in this storm?

Paulsen and colleagues ${ }^{8}$ have reported a bench-based model using a 3-dimensional-printed heart simulator designed to test the long-term efficacy of 2 routinely used aortic conduits for valve-sparing procedures. This is despite thousands of patients who have already received these 2 conduits. The novelty of this report is that it tries to address an important clinical question on the long-term durability of the conduits using a bench-based simulator. The simulator is ingenious, modeling physiological waveforms and mechanical activities of the heart. Porcine aortic root/valves were sutured in the 2 conduits followed by implanting silicone tubes as mock coronary buttons. A long shot.

At first look, few conflicting questions come to mind: is this simulator reliable? Can it really predict long-term durability by running it for few hours? Where is the rigorous confirmatory trial in large animals? Yet, we must respect this methodologic approach, as modern computational modeling has been shown to be effective in predicting health outcome. ${ }^{9}$ Based on the evidence arising from this simulation, the authors conclude that although the Valsalva graft accurately reflects the aortic root geometry longitudinally, its radial displacement of the valve commissures

triggers abnormal forces on the leaflets, affecting their long-term durability. A strong conclusion, with not a single patient operated on and not a drop of blood lost!

With the only variable in this simulator being the type of conduit used, it might be that the highlighted difference is genuine. Who knows? In a traditional translational research pathway, this would trigger an immediate need for in vivo validation in a relevant large animal model. However, the simulation has several limitations. The whole system looks too artificial as opposed to a typical in vivo model. The lack of vascular elasticity in the system is an issue. Suboptimal surgical expertise and/or mismatch between variable sizes of porcine valve used and the fixed diameter of the conduits selected might have affected the radial displacement of the leaflets. Finally, the simulation was conducted with normal saline solution and not with blood, using a fluid with different viscosity, hence affecting dynamics.

These are the practical immediate criticisms that we can raise. Translational large animal models with advanced longitudinal in vivo imaging would provide confirmatory knowledge of the pathophysiologic, mechanistic, and dynamic issues associated by this study with the Valsalva graft surgical procedure. This, in turn, would have more robust implications on the clinical scenario, possibly warranting a call for a comparative RCT? The study by Paulsen and colleagues ${ }^{8}$ also highlights that undertaking isolated benchbased simulation, no matter how good it can be, does not help much in moving the field forward. This is because real translation occurs across a pipeline in which bench 
simulation represents only the first step. This illustrates the key learning point that bench-based modeling needs to go hand-to-hand with advanced in vivo preclinical validation in relevant models ${ }^{10}$ to facilitate translation to bedside. Translating valuable basic science into patients requires time, funding, appropriate surgical skills, translational biomedical knowledge, and high experimental reproducibility. A gap in translation seems to be the main limitation of this process. Rigorous in vivo validation in relevant preclinical models may represent an effective way to bridge the gap between bench-based science and bedside across the storm. Like a bridge over troubled waters.

\section{References}

1. Drolet BC, Lorenzi NM. Translational research: understanding the continuum from bench to bedside. Transl Res. 2011;157:1-5.
2. Packer M. Are meta-analyses a form of medical fake news. Circulation. 2017; 136:2097-9.

3. Baker M. Is there a reproducibility crisis? Nature. 2016;533:452-4.

4. Who is not sharing their trial results? Available at: https://trialstracker. ebmdatalab.net/\#/. Accessed November 27, 2018.

5. Wadman M. A trans-Atlantic transparency gap on animal experiments. Science. 2017;357:119-20.

6. Alemzadeh H, Raman J, Leveson N, Kalbarczyk Z, Iyer RK. Adverse events in robotic surgery: a retrospective study of 14 years of FDA data. PLoS One. 2016; 104:1-20.

7. Medical device rules need "drastic change" to protect patients. Available at: https://www.bbc.co.uk/news/health-46337937. Accessed November 27, 2018

8. Paulsen MJ, Kasinpila P, Imbrie-Moore AM, Wang H, Hironaka CE, Koyano TK, et al. Modeling conduit choice for valve-sparing aortic root replacement on biomechanics with a 3D-printed heart simulator. J Thorac Cardiovasc Surg. 2019;158:392-403.

9. Morris PD, Narracott A, Von Tengg-Kobligk H, Silva Soto DA, Hsiao S, Lungu A, et al. Computational fluid dynamics modelling in cardiovascular medicine. Heart. 2016;102:18-28.

10. Doenst T, Schlensak C, Schibilsky D, Faerber G. Do we need basic research in cardiac surgery? Thorac Cardiovasc Surg. 2018;66:2-6. 